\title{
Influence of Mobile Network Interfering Products on DVB-T/H Broadcasting Services
}

\author{
Ladislav POLAK, Ondrej KALLER, Lukas KLOZAR, Jan PROKOPEC \\ Department of Radio Electronics, Brno University of Technology \\ Sensor, Information and Communication Systems (SIX) Research Center \\ Brno, Czech Republic \\ \{xpolak18,xkalle00,xkloza00\}@stud.feec.vutbr.cz,prokopec@feec.vutbr.cz
}

\begin{abstract}
In this paper the influence of mobile network interfering products on DTV (Digital Television) services is explored and evaluated. More precisely, the overall aim of this paper is to measure and show the interactions between the mobile (GSM) and digital terrestrial television (DVB-T/H) broadcasting networks and services. In the DVB-T/H system configuration, the TPS (Transmission Parameter Signaling) carriers keep the receiver informed about the main system parameters. Disturbance of these carriers by interfering products of GSM network and degradation of DVB-T services are also explored and described. Theoretical backgrounds, general workplace and the principle of the measuring method is also introduced and briefly described. For the evaluation of the impact of mobile networks on the signal quality of DTV services the BER (Bit Error Ratio), MER (Modulation Error Ratio) ratios and constellation imperfections are used. Finally, the obtained results are evaluated and clearly described.
\end{abstract}

Keywords-GSM; DVB-T/H; OFDM; TPS carriers; High order harmonic distortions; Interfering products; BER; MER;

\section{INTRODUCTION}

The use of wireless and mobile networks has expanded into the daily life of people and a life without services, provided by these networks, is unimaginable. Therefore, the increasing demand for stable wireless communication multimedia services is a key feature of advanced markets. In the context of this fact, of course, demand for mobile and DTT (Digital Terrestrial TV) reception of high quality video, audio and data broadcasting services are also rapidly increasing [1]. However, the best option to extend these services would be the digital dividend portion of the UHF spectrum (in this case from $790 \mathrm{MHz}$ to $862 \mathrm{MHz}$ ). Thanks to this, many bands are allocated for more than one multimedia service. Therefore, sharing frequency spectrums is inevitable and it will cause a high probability of interference of wireless systems with one another. Interference between these systems is called intersystem interference and occurs when these systems, operate at overlapping frequencies, share the same physical environment at the same time [2], [3].

On the last WRC-2007 (World Radio Conference) conference it was decided to allocate the $790-862 \mathrm{MHz}$ frequency band to mobile services in several world regions [1]. Due to this decision, the upper frequency band allocated for the DVB-T system in Europe $(606-854 \mathrm{MHz})$ is co-allocated to mobile services. Therefore, this should cause undesired mutual interferences between mobile services and DTT that needs to be carefully analyzed. It is important because GSM and DVB$\mathrm{T} / \mathrm{H}$ technologies are mainly used in Europe and in parts all over the world.[4].

The GSM (Global System for Mobile Communication) system is the most widely used cellular technology in the world today and it is originally described as a digital network, optimized for full duplex voice telephony. GSM services are operating within the existing UHF spectrum, in Europe especially from $880 \mathrm{MHz}$ to $960 \mathrm{MHz}$ with $200 \mathrm{kHz}$ wide channel bandwidth. On the other hand, DVB-T/H is a classic standard and technology for digital terrestrial TV distribution. The DVB-T/H system services are operating within the existing UHF $(470-870 \mathrm{MHz})$ spectrum, in TV channels from C22 to C61 [5], [6].

Of course, unaware interference caused by various wireless systems operating in the same or neighboring frequency bands, is not a new phenomenon and it is becoming one of the most important issues. Currently, this field is still being explored, because the co-existing wireless networks can suffer significant mutual interference and performance degradation. In literature many studies can be found which deal with this issue. In [7], e.g., the authors investigated the co-existence issues between macro/micro WCDMA (Wideband Code Division Multiple Access) and WIMAX macro systems. Furthermore, the intra system and inter system interference model has also been studied, when a very perspective LTE (Long Term Evolution) and classical WIMAX coexist [8]. In [10] and [11], the authors were testing the interferences influences on the DVB-T signals transmitted over a simulated AWGN (Gaussian) channel. On the other hand, in [12] and [13], signal distortions on the DVB$\mathrm{T}$ system caused by GSM, UMTS (Universal Mobile Telecommunications System) and LTE are explored.

From the point of industrial applications, it can be seen that interference, as a product of mutual co-existence of different multimedia technologies, is the most critical issue. In our last work [12], we explored the interactions between mobile and DVB-T broadcasting services. In this work and progress paper, our investigation is focused on exploring the influence of GSM network interfering production DVB-T/H services. For this purpose, the workplace presented and used in [12] was slightly modified. Furthermore, the general workplace for the automated measuring of typical DVB-T/H amplifier

This paper was supported by the project of Ministry of Education, Youth and Sports no. CZ.1.07/2.3.00/20.0007 (WICOMT), national project no. LD12005 "Quality of Experience aspects of broadcast and broadband multimedia services (QUALEXAM)" and LD11081 "System of Wireless Internet Communication (SYWIC)" in the COST IC0906 WiNeMO action, ENIAC JU project no. 270683-2 and MEYS of the Czech Republic project no. 7H11097 "Agile RF Transceivers and Front-Ends for Future Smart Multi-Standard Communications Applications (ARTEMOS)"and BUT university internal grant project FEKT-S11-12. The research was performed in laboratories supported by the SIX project; no. CZ.1.05/2.1.00/03.0072. 
nonlinearity was realized and used. The paper is organized as follows: The measurement setup of nonlinearity of the DVB$\mathrm{T} / \mathrm{H}$ amplifier is presented in Section II. A brief description of the TPS carriers and the method of them being affected by interfering products is outlined in Section III. Section IV introduces the measurement workplace used for measuring the influence of GSM interfering products on DVB services. The experimental results, obtained from the measurements, are also evaluated and discussed in section IV. Finally, the paper concludes in Section V.

\section{TYPICAL DVB-T/H AMPLIFIER NONLINEARITY}

To investigate crosstalk and interference scenarios between DTV broadcasting and mobile standards, we should identify the main sources of these phenomena. One of them and perhaps the most widespread is the television (pre)amplifier. In the case of a central television antenna system, narrowband high directional antennas are often used and also the amplifiers have built-in filters for each band. Fortunately, the input filters in back-propagation of higher harmonic components are averted.

Another situation occurs in the case of individual TV reception. Indoor omni-directional antenna with a broadband amplifier is typically used. Unfortunately, these are easily overloaded and moreover, the output signal can saturate the input part of the TV tuner. These produce high order components which are easily emitted by the antenna as interfering products. In indoor location, these facilities are set up by laic users. Therefore, we have to expect besides strong downlink, also amplified uplink signals of different mobile standards.

Preliminary system modeling has been considered to describe typical amplifier nonlinearity by a power of higher harmonic components, not by typical models such as AM/AM, AM/FM or SALEH. Models without memory do not provide enough accuracy. Of course, implementation of advanced models will be done later. A wiring diagram of the laboratory facility is shown in Fig 1.

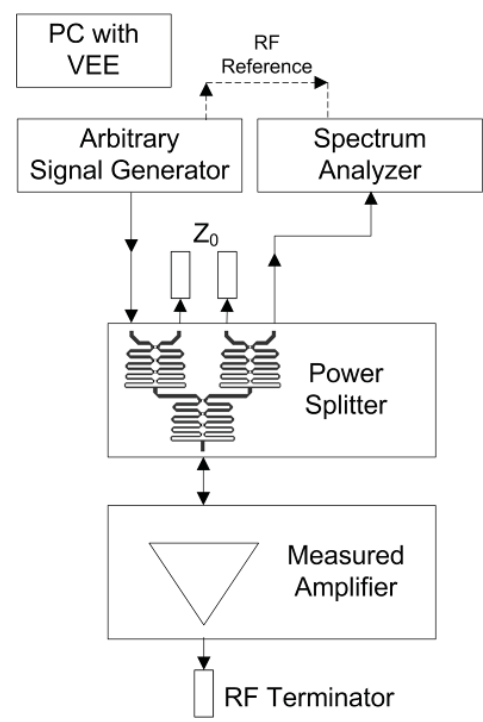

Figure 1. Block diagram used for amplifier nonlinearity measurement.

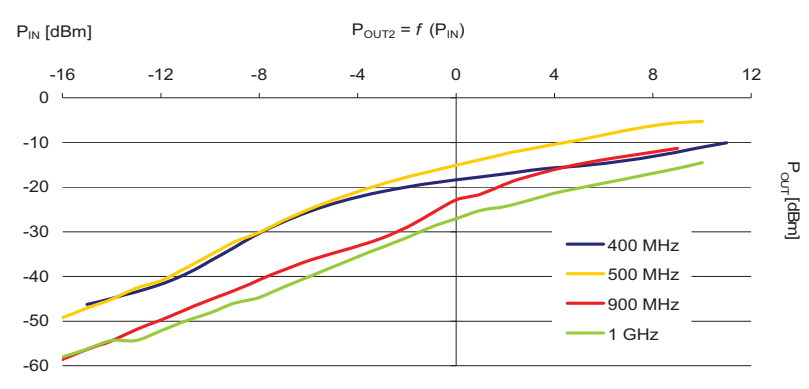

Figure 2. Output level of second harmonic component dependency on input signal level in the case of three input signal frequencies.

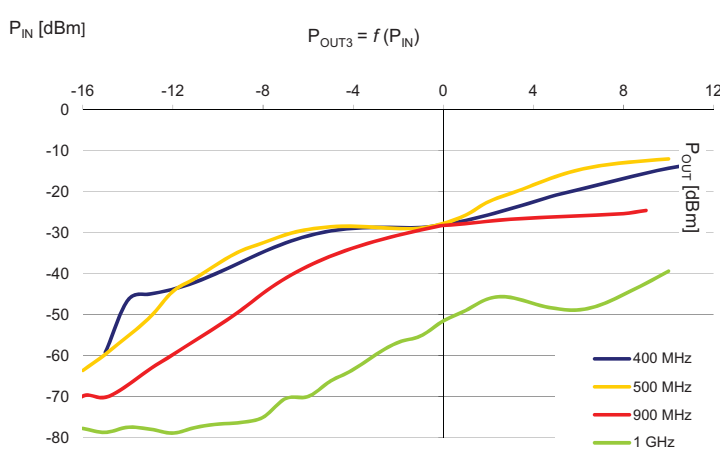

Figure 3. Output level of the third harmonic component dependency on the input signal level in the case of three input signal frequencies.

Output impedance matched amplifier is fed by one GSM channel $(200 \mathrm{kHz})$ by signal from arbitrary generator. Unwanted output product level is measured using a spectrum analyzer. Due to insufficient isolation between the output of generator and analyzer, the SWR (Standing Wave Ratio) can not be measured. Therefore, the output level of the $2^{\text {nd }}$ to $6^{\text {th }}$ harmonic component has been measured at the input amplifier port. The most significant ones, the $2^{\text {nd }}$ and $3^{\text {rd }}$, are shown in Fig. 2 and 3.

\section{STRUCTURE OF TPS CARRIERS IN DVB-T/H STANDARD}

DVB-T is the DVB European standard for broadcasting audio, video and data TV services over an MPEG-2 stream using OFDM (Orthogonal Frequency Division Multiplexing) modulation. The OFDM transmitted signal is organized in frames and each of them consist of 68 OFDM symbols. Each symbol consists of $K$ subcarriers. Depending on the OFDM mode, used in DVB-T ( $2 \mathrm{~K}$ or $8 \mathrm{~K}$ ), the $K$ equals 1705 (2K) or 6817 (8K) [5], [6].

All symbols in an OFDM frame contain data and reference information. Moreover, each OFDM frame contains special types of subcarriers. These pilot signals are used for automatic frequency control (continual pilots), for channel estimation and correction (scattered pilots) and transmitting system information from the transmitter to the receiver (TPS Transmission Parameter Signalling carriers) [5], [6]. In this paper, we are mainly focusing on TPS carriers, because these carriers contain the most important system parameters for the receiver, as will be briefly outlined below. 
TPS carriers are used for the purpose of signaling parameters related to the transmission scheme. These carriers keep the receiver informed about modulation, inner code rates, OFDM mode and frame number. The complete list can be found in [6]. Each OFDM frame conveys one TPS bit. Each TSP, corresponding to one OFDM frame, contains 68 bits, defined as initialization, information, synchronization and redundancy bits. More usable information can be found in [5] and [6].

TPS carriers contain important system parameters for the receiver. Due to this, we can expect that when these carriers are influenced by interfering products (e.g. from mobile networks), then the receiver will have problems with synchronization and with processing a received TV signal. Therefore, exploring the impact of interfering products on TPS carriers is one of the main purposes of this paper. For analyzing the affect of TPS carriers in each OFDM symbol, it is important to determine the exact frequency where these carriers are working. The general formula for calculating the $N^{\text {th }}$ TPS carrier is defined in equation (1):

$$
f_{N}=f_{C}-\frac{B W_{C}}{2}+\frac{1}{\left(B W_{T V}-N o_{S C}\right) T} \cdot N,
$$

where $f_{N}$ is the frequency of the $N^{\text {th }}$ carrier, $f_{C}$ is the carrier frequency of the DVB-T signal $(778 \mathrm{MHz}), B W_{T V}$ is the TV channel bandwidth $(8 \mathrm{MHz}), B W_{C}$ is the spacing between the first and the last OFDM carrier, $N o_{S C}$ is the total number of all subcarriers in the DVB-T channel (8192 for 8K OFDM mode), $T$ is the elementary period $T=7 / 48 \mu s$ and $N$ is the number of the actual OFDM carrier.

\section{EXPERIMENTAL MEASUREMENT AND RESUlts}

In this part, the measurement workplace and its setup, used in this paper, are introduced. We focus on the interferences generated by the co-existence of mobile and television wireless standards in adjacent frequency bands. This measurement quantifies the influence of the generated interfering products overlaying the tested DVB-T channel.

\section{A. Conception}

The generated interfering signals, originating from mobile networks, are assumed as GSM and/or LTE signals. The interfered DVB-T signal is generated at a frequency of $778 \mathrm{MHz}$. It occupies an $8 \mathrm{MHz}$ wide channel, works in $8 \mathrm{~K}$ OFDM mode and uses 64QAM modulation. We try to test how the level of overlaying interfering products affects the quality of broadcasting services with various FEC (Forward Error Correction) code rates. Therefore, our experiment consists of two parts.

In the first part we generate the bandpass signals to simulate mobile network interferences. The narrowest interfering signal is $200 \mathrm{kHz}$ wide and corresponds to the $1^{\text {st }}$ harmonic of a GSM channel. The widest one has $4 \mathrm{MHz}$ bandwidth (to overlay half of the DVB-T channel). Interfering signals are positioned on the center of the DVB-T channel (carrier frequency is equal to $778 \mathrm{MHz}$ ).

In the second part we have focused on the TPS reference signals of DVB-T. For the interfere just the desired OFDM subcarriers in TV channel we use generated signal with bandwidth approx. $1 \mathrm{kHz}$, which correspond to the subcarrier bandwidth, used in DVB-T 8K mode [6]. Furthermore, we concatenated exact number of carriers to overlay the TPS subcarrier frequencies, according to (1). We also aggregated such a number of interfering sub signals to satisfy the specified channel bandwidth of interfering bandpass signals.

We have also interfered all 68 TPS subcarriers at once and observed the influence on the quality of received TV signal. The purpose was to explore impact of the affected TPS carriers on the valid signal reception and synchronization.

\section{B. Measurement Setup and Approach}

General principle of the simplified measuring method for the interactions between mobile and DVB services is shown in the block diagram in the Fig. 4.

Interfering bandpass signals are produced in $\mathrm{R} \& \mathrm{~S}$ WinIQSIM2 software, using RRCF (Root-Raised Cosine Filter) with the roll-of factor 0.35 . We have generated eleven signals with different bandwidth. To involve possible interferences, originated from the GSM network, the first, second and third harmonic (200, 400 and $800 \mathrm{kHz})$ of GSM channel was taken into account. For the LTE system the first and the second harmonic of $1.4 \mathrm{MHz}$ channel and the first harmonic of $3 \mathrm{MHz}$ channel were tested. Interfering signals of bandwidths $1,2,2.4,3.5$ and $4 \mathrm{MHz}$ were also included to uniformly cover desired bandwidth (up to $4 \mathrm{MHz}$ ).

Interfering signals for TPS scenario were chosen with same bandwidths as bandpass signals, except the narrow signals (200, $400 \mathrm{kHz}$ bandwidths were excluded for the low number of overlaying TPS). Also the $1 \mathrm{MHz}$ wide signal was excluded.

The measuring technique consist in keeping the constant level of the DVB-T/H signal and increasing the level of the interfering signal (to satisfy the minimum receiver input level $-76.9 \mathrm{dBm}$ of the DVB-T/H receiver, defined in [14], for $8 \mathrm{~K}$, 64QAM and $2 / 3$ code rate). The standardized level on TV signal was equaled to $50 \mathrm{~dB} \mu \mathrm{V}(-50.8 \mathrm{dBm})$ and we set the level on the value $48 \mathrm{~d} \mu \mathrm{BV}$ that corresponds with $-60.8 \mathrm{dBm}$.

The measurement was performed for the most used FEC code rates of the DVB-T/H standard (2/3, 3/4, 5/6 and 7/8). To evaluate the quality of services we used two criterions. The first one is the QEF (Quasi Error-Free) operation [5], [6] as BER after Viterbi decoding less or equal to $2.10^{-4}$. The second one is the subjective image criterion based on SSIM (Structural Similarity). The limit for the sufficient subjective video quality was set as the value of SSIM, equal to $50 \%$. We used a standard video sequence (FACT 9M.gts), compressed by MPEG-2 standard with data rate $9 \overline{\mathrm{Mb}} \mathrm{s}$. The test signals, produced in R\&S WinIQSIM2, were loaded and generated in signal generator and compound with the DVB-T/H signal.

The realized laboratory workplace (see Fig. 5) in the Laboratory of Mobile Communication Systems, Brno University of Technology has been used for the measuring of the influence of mobile network interfering products on DVB-T/H broadcasting services.

\section{Measurement Results}

Compound signals (interfered and interfering) were analyzed on the DVB-T/H measurement receiver and spectrum analyzer (see Fig. 5). 


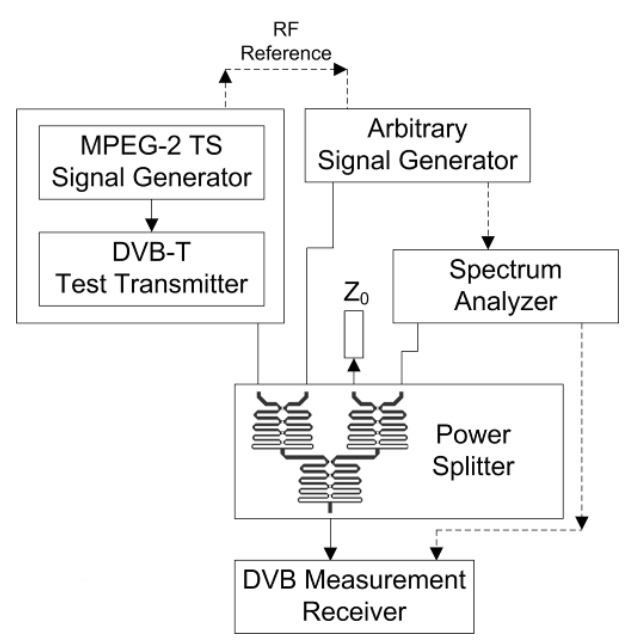

Figure 4. Block diagram of workplace for the measurement of the interaction between GSM, LTE and DVB-T/H services.

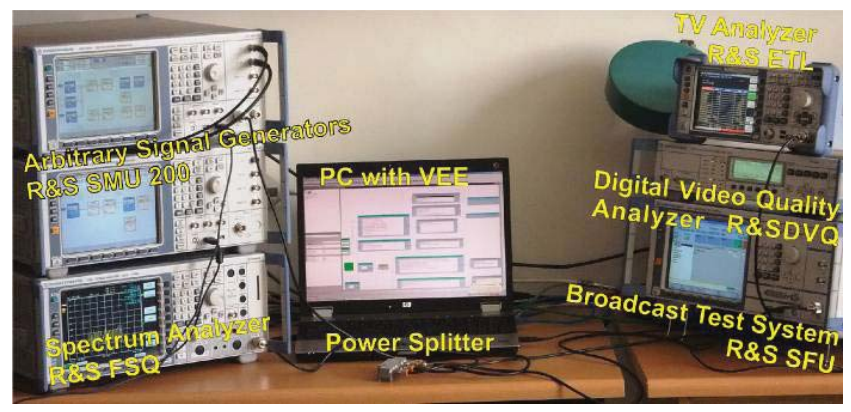

Figure 5. Laboratory workplace for the measuring of the interaction between mobile and digital terrestrial television broadcasting networks (GSM and LTE generators, SFL-T DVB-T/H transmitter, DVB-T/H test receiver and analyzer and spectral analyzer).

Measurement results for bandpass interferences are summarized in Fig. 6 a) to b). The DVB-T/H signal to interfering signal ratio expresses the amount of interferences at which the valid reception is achievable. It is depend on the used FEC code rate and on the width of interfering signal. As it was mentioned above, there were used two criterions for the evaluating of the obtained results.

Firstly, the QEF reception was explored, when the digital TV signal to interfering signal ratio is depending on the bandwidth of the interfering signal (see Fig. 6 a)). Of course, the measurement was done for the most used FEC code rates, used in the DVB-T/H standard. From the obtained result is clearly seen that when the bandwidth of the interfering signal is higher then the interfering ratio is also higher and the level of the DVB-T/H signal is decreasing. The worst results were obtained at code rate $7 / 8$ (lowest error protection). Secondly, the subjective image quality was investigated (see Fig. 6 b)). Obtained results, compare to Fig. 3 a), are slightly different.

Dependences of the DVB-T/H signal to interfering signal ration on the bandwidth of the interfering signal for dedicated TPS interfering for different FEC code rates are shown in Fig. 7 a) to b).

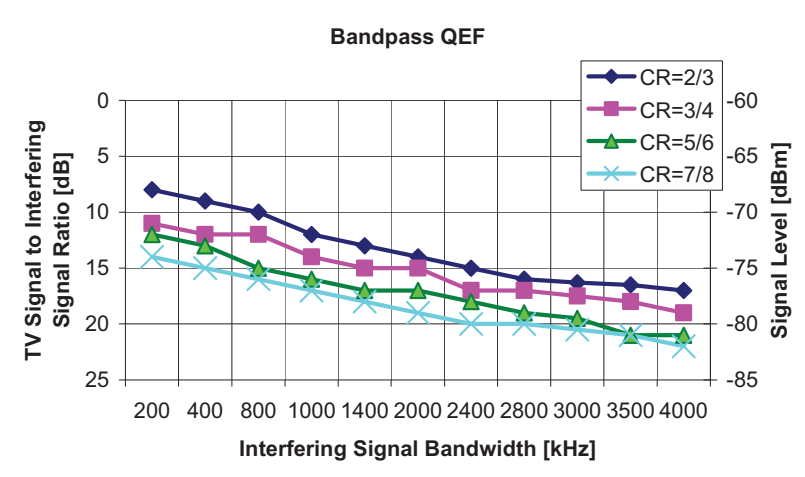

a) Bandpass QEF

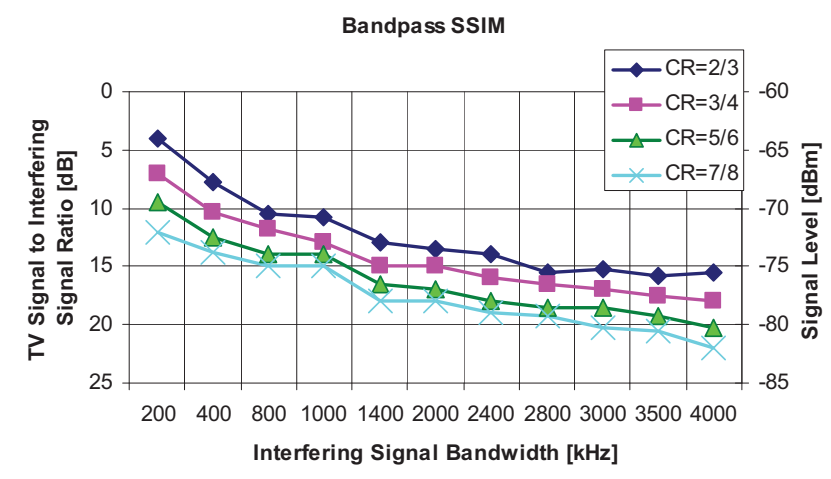

b) Bandpass SSIM

Figure 6. Code rate dependency of the TV signal to interfering signal ratio on interfering signal bandwidth for bandpass interfeering signals. The level of the TV signal is equal to $-60 \mathrm{dBm}$.

The number of interfered TPS carriers is depending on the bandwidth of the interfering signal. Of course, this dependence has also impact on the received TV signal quality. And again, the QEF operation and the SSIM method used for the evolution of the results.

In the case of the QEF reception, again, the best signal level at can be achieved only when the $2 / 3$ code rate is used. The signal level between the two critical interfering signal bandwidth $(0.8 \mathrm{MHz}$ and $4 \mathrm{MHz})$ is approx. the same. The $2 / 3$ code rate is the exception, where between the $0.8 \mathrm{MHz}$ and $1.4 \mathrm{MHz}$ is higher difference (see Fig. 7 a)). Surprisingly, for the other code rates $(3 / 4,5 / 6$ and $7 / 8)$, the obtained results and their graphical form are very similar. In the case, when the SSIM method is used (see Fig. 7.b)), the obtained result are again very similar, compare to results, which are obtained in case of QEF reception. In this case, the distance between the $2 / 3$ and other FEC code rates is more significant.

As a last one, we also explored the scenario, when all (68) TPS carriers are interfered. Obtained results (only for the most used DVB-T/H code rate $-2 / 3$ ) for both criterions (QEF and SSIM), used in this paper, are clearly presented in Tab I. 


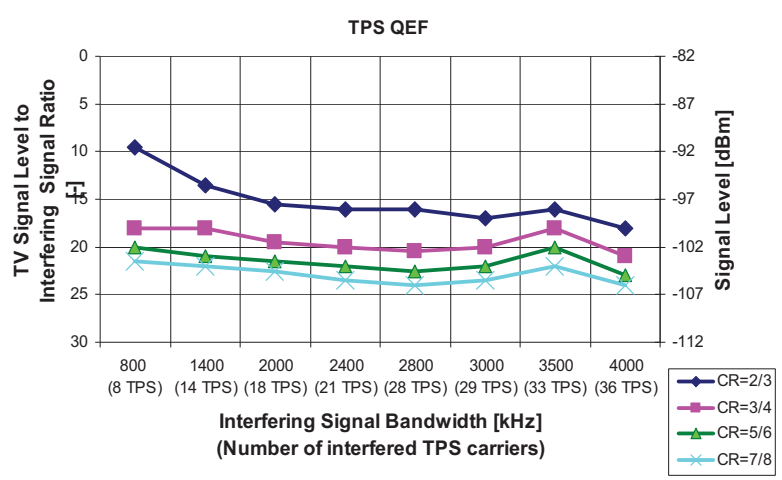

a) TPS QEF

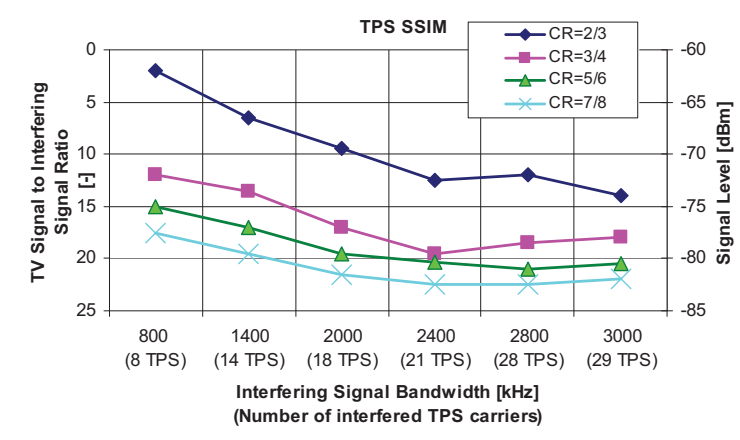

b) TPS SSIM

Figure 7. Code rate dependency of the TV signal to interfering signal ratio on interfering signal bandwidth for dedicated TPS interfeering signals. The level of the TV signal is equal to $-82 \mathrm{dBm}$.

TABLE I. MEASURED VALUES FOR 68 INTERFERED TPS CARRIERS

\begin{tabular}{|c|c|c|c|}
\hline \multirow[b]{2}{*}{ Criterion } & \multicolumn{3}{|c|}{ Measured Values } \\
\hline & $\begin{array}{c}\text { TPS } \\
\text { LEVEL } \\
{[d B m]}\end{array}$ & $\begin{array}{c}T V \\
L E V E L \\
{[d B m]}\end{array}$ & $\begin{array}{c}\text { TV to TPS Ratio } \\
\text { [dB] }\end{array}$ \\
\hline $\begin{array}{l}\text { QEF (BER after Viterbi } \\
\text { is less or equal to } 2 \cdot 10^{-4} \text { ) }\end{array}$ & -102 & -82 & 20 \\
\hline SSIM $>50 \%$ & -98 & -82 & 16 \\
\hline
\end{tabular}

\section{CONCLUSIONS}

In this paper the influence of mobile network interfering products on DVB-T/H services was explored and evaluated. Our experiment was divided into two parts. Firstly, we explored the effect of mobile network interferences. For this purpose we generated bandbpass signals with different bandwidth. Secondly, our attention was focused on TPS carriers used in the DVB-T/H standard. More precisely, to interfere only the desired OFDM subcarriers in the fixed TV channel. To evaluate the quality of services two criteria were used: QEF operation and the SSIM method. From the obtained results it is easy to see that the interactions between the mobile and DVB-T/H services really does exist and it must be investigated in the future more thoroughly.

This work will continue by finishing and improving the proposed method for measuring the interactions between mobile (GSM, UMTS and LTE) and DVB-T/H/T2 services. Future work will also be focused on creating an appropriate application (database), which will enable to determine potential scenarios (based on the frequencies of different services and the level of their signals), when interactions between mobile and DVB services occur. Moreover, we are also considering extending our research with real transmission scenarios [4], [11], [15], [16].

\section{REFERENCES}

[1] A. Aloisi, M. Celindonio, L. Pulcini and A. Rufini, "A simulation study of broadband mobile systems interference on DVB-T systems operating in the UHF frequency band," in 2011 Future Network \& Mobile Summit. Warsaw (Poland), 2011, p. 1 - 8.

[2] Z.A. Shamsan and T..A. Rahman, "Intersystem interference scenarios between fixed and IMT-Advanced services in different terrestrial regions," in Proc. of the 2009 International Conference on Future Computer and Communication ICFCC 2009. Kuala Lumpur (Malaysia), 2009, p. 237-240.

[3] Z.A. Shamsan and T..A. Rahman, "IMT-coexistence method with fixed services in different geographical area at $3500 \mathrm{MHz}$," in Proc. of the IEEE EUROCON 2009, p. 1528-1533.

[4] K. Šakić, M. Gosta and S. Grgić, „Cross-border interference between broadcasting and mobile services," In Proc. of the $51^{\text {st }}$ International Symposium ELMAR 2009. Zadar (Croatia), 2009, p. 229-232.

[5] W. Fisher, Digital Video and Audio Broadcasting Technology. A Practical Engineering Guide. $2^{\text {nd }}$ ed. Springer, 2008.

[6] ETSI EN 300744 V1.6.1 (2009-01). Digital Video Broadcasting (DVB), Framing structure, channel coding and modulation for digital terrestrial television. European Standard ETSI, 2009.

[7] G. Peng, T. Guofang, F. Yuan and L. Shuangchun, "The analysis of the interferences between WCDMA and WIMAX systems," in Proc. of the International Conference on Coomunications Technology and Applications- ICCTA 2009. Beijing (China), 2009, p. 180-184.

[8] Y. Zang, H. Guo, W. Xinglin and Z. Xiaojin, "Co-existence analysis of LTE and WIMAX in uplink," in Proc. of the $6^{\text {th }}$ International Conference on Wireless Communications, Networking and Mobile Computing- WICOM 2010. Chengu (China), 2010, p. 1-4.

[9] A. Lourerio, D. Gallegos, D. and G. Caldwell, "Interference analysis on UMTS-2100 co-existence with GSM-1900," In Proc. of the 2011 IEEE Vehicular Technology Conference Fall - VTC Fall 2011. San Francisco (California), 2011 p. 1-4.

[10] T. Kratochvil. and V. Ricny, "Simulated testing of the interferences influence on the DVB-T signal," in Proc. of the $19^{\text {th }}$ International Conference Radioelektronika 2009. Bratislava (Slovakia), 2009, p. 107110.

[11] A. Guidotti, et. all, "Coexistence and mutual interference between mobile and broadcasting systems," in Proc. of the $73^{\text {rd }}$ IEEE Vehicular Technology Conference - VTC 2011. Budapest (Hungary), 2011, p.1-5.

[12] L. Polak, O. Kaller, L. Klozar and J. Prokopec, "Measuring of the interactions between mobile and DVB-T broadcasting networks," in Proc. of the $22^{\text {nd }}$ International Conference Radioelektronika 2012. Brno (Czech Republic), 2012, p. 243-246.

[13] K. Šakić and S. Grgić, "The Influence of the LTE system on DVB-T reception," in Proc. of the 52 ${ }^{\text {nd }}$ International Symposium ELMAR 2010. Zadar (Croatia), 2010, p. 235-238.

[14] IEC 62216-1 First edition (2001-10). Digital terrestrial television receivers for the DVB-T system - Part 1: Baseline receiver specification. International Standard, 2001.

[15] L. Polak and T. Kratochvil, "DVB-H and DVB-SH-A performance and evaluation of transmission in fading channels ," in Proc. of the $34^{\text {th }}$ International Conference on TSP 2011. Budapest (Hungary), 2011, p. 549-553.

[16] L. Polak and T. Kratochvil, "DVB-T and DVB-T2 performance in fixed terrestrial TV channels," in Proc. of the $35^{\text {th }}$ International Conference on TSP 2012. Prague (Czech Republic), 2012, p. 725-729. 